\title{
PREP1 deficiency downregulates hepatic lipogenesis and attenuates steatohepatitis in mice
}

\author{
Francesco Oriente - Serena Cabaro • Antonietta Liotti • Michele Longo • Luca Parrillo • \\ Teresa Bruna Pagano • Gregory Alexander Raciti • Dmitry Penkov • Orlando Paciello • \\ Claudia Miele • Pietro Formisano • Francesco Blasi • Francesco Beguinot
}

Received: 19 June 2013 / Accepted: 26 August 2013 /Published online: 20 September 2013

(C) Springer-Verlag Berlin Heidelberg 2013

\begin{abstract}
Aims/hypothesis The aim of this study was to investigate the function of Prepl (also known as Pknoxl) in hepatic lipogenesis. Methods The hepatic lipogenesis pathway was evaluated by real-time RT-PCR and Western blot. Biochemical variables were assessed using a clinical chemistry analyser.

Results Serum triacylglycerols and liver expression of fatty acid synthase (FAS) were significantly decreased in Prep1 hypomorphic heterozygous (Prep $1^{i / 4}$ ) mice compared with their non-hypomorphic littermates. Upstream FAS expression, phosphorylation of protein kinase $\mathrm{C}(\mathrm{PKC}) \zeta$, liver kinase $\mathrm{B} 1$ (LKB1), AMP-activated protein kinase (AMPK) and acetylCoA carboxylase (ACC) increased in Prep $1^{i /+}$ mice, while protein and mRNA levels of the lipid phosphatase inhibitor of PKCद, SH2-containing inositol 5'-phosphatase 2 (SHIP2), was more than $60 \%$ reduced. Consistent with these findings, HepG2 cells transfected with Prepl cDNA exhibited
\end{abstract}

Francesco Oriente and Serena Cabaro contributed equally to this study.

Electronic supplementary material The online version of this article (doi:10.1007/s00125-013-3053-3) contains peer-reviewed but unedited supplementary material, which is available to authorised users.

F. Oriente $\cdot \mathrm{S}$. Cabaro $\cdot$ A. Liotti $\cdot$ M. Longo $\cdot$ L. Parrillo

G. A. Raciti $\cdot$ C. Miele $\cdot$ P. Formisano $\cdot$ F. Beguinot $(\square)$

Department of Translational Medical Sciences, 'Federico II'

University of Naples and Institute of Experimental Endocrinology

and Oncology, National Council of Research,

Via Pansini 5, 80131 Naples, Italy

e-mail: beguino@unina.it

T. B. Pagano $\cdot$ O. Paciello

Department of Veterinary Medicine and Animal Production,

'Federico II' University of Naples, Naples, Italy

D. Penkov $\cdot$ F. Blasi

IFOM (FIRC Institute of Molecular Oncology), Milan, Italy

D. Penkov

Moscow State University, Moscow, Russia increased triacylglycerol accumulation and FAS expression, with strongly reduced PKC $\zeta$, LKB1, AMPK and ACC phosphorylation. Further experiments revealed the presence of both Prep1 and its major partner Pbxl at the Ship2 (also known as Inppl1) promoter. PBX-regulating protein 1 (PREP1) and pre-B cell leukaemia transcription factor 1 (PBX1) enhanced Ship2 transcription. The PREP $1_{\mathrm{HR}}$ mutant, which is unable to bind PBX1, exhibited no effect on Ship2 function, indicating transcriptional activation of Ship2 by the PREP1/PBX1 complex. Treatment with a methionine- and choline-deficient diet (MCDD) induced steatosis in both Prep $1^{i / 4}$ and non-hypomorphic control mice. However, alanine aminotransferase increase, intracellular triacylglycerol content and histological evidence of liver steatosis, inflammation and necrosis were significantly less evident in Prep $1^{i /+}$ mice, indicating that Prep1 silencing protects mice from MCDD-induced steatohepatitis.

Conclusions/interpretation Our results indicate that Prep1 silencing reduces lipotoxicity by increasing PKC ¿/LKB1/ AMPK/ACC signalling, while levels of PREP1 expression may determine the risk of steatohepatitis and its progression.

Keywords AMPK · Lipogenesis · PBX1 - PREP1 · SHIP2 · Steatohepatitis

$\begin{array}{ll}\text { Abbreviations } \\ \text { ACC } & \text { Acetyl-CoA carboxylase } \\ \text { ALT } & \text { Alanine aminotransferase } \\ \text { AMPK } & \text { AMP-activated protein kinase } \\ \text { CAMKK } \beta & \text { Calcium/calmodulin-dependent protein kinase } \\ & \text { kinase } \beta \\ \text { ChIP } & \text { Chromatin immunoprecipitation } \\ \text { FAS } & \text { Fatty acid synthase } \\ \text { HepG2 } & \text { Human hepatocellular carcinoma } \\ \text { LKB1 } & \text { Liver kinase B1 } \\ \text { MCDD } & \text { Methionine- and choline-deficient diet }\end{array}$




$\begin{array}{ll}\text { NAFLD } & \text { Non-alcoholic fatty liver disease } \\ \text { NASH } & \text { Non-alcoholic steatohepatitis } \\ \text { ORO } & \text { Oil Red O } \\ \text { PBX } & \text { Pre-B cell leukaemia transcription factor } \\ \text { PKA } & \text { Protein kinase A } \\ \text { PKC } & \text { Protein kinase C } \\ \text { PREP1 } & \text { PBX-regulating protein 1 } \\ \text { SHIP2 } & \text { SH2-containing inositol 5'-phosphatase 2 } \\ \text { SREBP } & \text { Sterol regulatory element binding protein } \\ \text { TG } & \text { Triacylglycerol } \\ \text { WT } & \text { Wild-type }\end{array}$

\section{Introduction}

The liver has a very important role in lipid homeostasis and metabolism [1]. In the liver, an excess of carbohydrates results in insulin-dependent de novo fatty acid synthesis. This effect is largely accomplished through the downregulation of AMPactivated protein kinase (AMPK) [2]. Indeed, AMPK phosphorylates and inactivates acetyl-CoA carboxylase (ACC), inhibiting fatty acid biosynthesis [3, 4]. AMPK activation requires phosphorylation at Thr172 by one or more upstream kinases, including liver kinase B1 (LKB1) [5, 6].

In addition to the de novo synthesis of fatty acids, however, other events can cause the intrahepatic accumulation of lipids, including increased lipid uptake, impaired lipoprotein secretion or synthesis and reduced fatty acid oxidation [7]. Insulin resistance can impact on several of these processes, leading to excess liver fat deposition and non-alcoholic fatty liver disease (NAFLD). NAFLD, in turn, facilitates oxidative hepatocellular damage, inflammation and activation of fibrogenesis. The resulting non-alcoholic steatohepatitis (NASH) can progress towards cirrhosis and hepatocellular carcinoma. NAFLD represents the most common liver disease and the leading cause of altered liver enzymes in Western countries [8]. Its development parallels that of insulin resistance and it is associated with type 2 diabetes mellitus [9].

Epidemiological, familial and twin studies provide strong evidence for NAFLD heritability [10, 11]. Genetic modifiers of disease severity and progression have been identified through genome-wide association studies [8, 12, 13]. Furthermore, a few large multicentre studies have demonstrated the role of gene variants implicated in insulin signalling, oxidative stress and fibrogenesis in the progression of NAFLD towards NASH and fibrosis [12,14]. These studies also confirmed the significance of intrahepatocyte fat accumulation and systemic insulin resistance for the progression of liver damage. However, the genetic bases of NAFLD and NASH risk and the relationship with insulin resistance are far from clear.

Pre-B cell leukaemia transcription factor-regulating protein 1 (PREP1) is a homeodomain transcription factor belonging to the TALE family of proteins. This family of proteins also includes the PBX (PBX1-4) and MEIS (MEIS1-3) transcription factors, and plays an important role in haematopoiesis, organogenesis and development. Both PREP and MEIS contain homologous conserved PBX-interacting motifs (HR1 and HR2 domains) and form transcriptionally active complexes with PBX [15-17]. However, while PREP1's main interactor is PBX1, MEIS1 is most involved in interactions with HOX proteins; likewise, while PREP1 acts mainly at promoter sites, MEIS1 acts mainly at promoter-distant sites [18]. As complete Prepl (also known as Pknoxl) knockout is lethal, previous work has shown that Prepl hypomorphic heterozygous $\left(\right.$ Prep $\left.^{i / 4}\right)$ mice feature a complex phenotype characterised by increased insulin sensitivity and protection from streptozotocin-induced diabetes [19]. These animals also feature decreased hepatic triacylglycerol (TG) content, raising the hypothesis that Prep1 may impact not only on glucose, but also on lipid metabolism in the hepatocyte [20].

In this work, we have addressed this issue and investigated the function of Prepl on fatty acid metabolism and TG accumulation in liver. We show that the PREP1/PBX1 complex upregulates transcription of the lipid phosphatase Ship2 (also known as Inppl1), thereby controlling the protein kinase $\mathrm{C}$ (PKC) $/ \mathrm{LKB} 1 / \mathrm{AMPK} / \mathrm{ACC}$ signalling cascade, fatty acid synthase (FAS) expression and TG accumulation in liver cells. On the basis of these results, Prep1 might be considered a new gene involved both in the pathogenesis of insulin resistance and steatohepatitis.

\section{Methods}

Materials Media, sera, antibiotics for cell culture and the Lipofectamine reagent were all from Invitrogen (Grand Island, NY, USA). The $p R C / C M V$-Prepl, $p S G 5-P b x 1$ and PSG5-Prep $1_{H R I}$ vectors have been previously described [20, 21]. PREP1, actin, PBX1, SH2-containing inositol 5'-phosphatase 2 (SHIP2), pACC, ACC, AMPK, LKB1, calcium/ calmodulin-dependent protein kinase kinase $\beta$ (CAMKK $\beta)$, pPKC $\zeta, \mathrm{PKC} \zeta$, phosphorylated protein kinase A (pPKA) and PKA antibodies were from Santa Cruz Biotechnology (Santa Cruz, CA, USA). pLKB1, pCAMKK $\beta$ and pAMPK antibodies were from Cell Signaling Technology (Beverly, MA, USA). Protein electrophoresis and real-time PCR reagents were from Bio-Rad (Richmond, VA, USA). Western blotting and ECL reagents were from Amersham Biosciences (Arlington Heights, IL, USA). Oil Red O (ORO) stain and all other chemicals were from Sigma (St Louis, MO, USA).

Studies in mice Prep 1 -targeted mice were generated by gene trapping by Lexicon Genetics (The Woodlands, TX, USA). The general phenotype of these mice has been previously described [21-23]. In the experiments reported in this paper, Prep $1^{i /+}$ mice were backcrossed with wild-type (WT) 
C57BL/6J mice for generations. All animal handling conformed to regulations of the Ethics Committee on Animal Use of H. S. Raffaele (IACUC permission number 207). The genotyping strategy has been previously described [19].

Hepatic tissue samples were collected rapidly from 8- to 10-week-old male C57BL/6-SV129 mice. Tissues were snapfrozen in liquid nitrogen and stored at $-80^{\circ} \mathrm{C}$ for subsequent western blotting and real-time RT-PCR analysis, as previously described [19].

The blood for serum biochemistry evaluation was collected with an intracardiac method, allowed to clot at room temperature and centrifuged for $10 \mathrm{~min}$; the serum was then separated for shipment. Quantitative analysis of serum TGs was performed with an ABX Pentra400 clinical chemistry analyser using the reagent ABX Pentra Triacylglycerols CP (ABXHoriba, Montpellier, France) according to the manufacturer's instructions. Serum VLDL-cholesterol and -triacylglycerol levels were measured with ELISA kits (USCN Life Science, Houston, TX, USA).

Methionine- and choline-deficient diet Nine-week-old male C57BL/6-SV129 mice (WT and Prep ${ }^{i / 4}$ ) were divided into four groups. Two groups of mice $(n=9 \mathrm{WT}$ and $n=9$ Prep $\left.1^{i /+}\right)$ were fed a standard rodent chow diet. The other two groups of mice ( $n=9 \mathrm{WT}$ and $n=9$ Prep $^{i /+}$ ) were fed a methionine- and choline-deficient diet (MCDD). All of the animals were maintained in a temperature- and lightcontrolled facility, and permitted ad libitum consumption of food and water throughout the 4 weeks of the experimental period. Body weights were measured every week. The animals were killed after overnight fasting for tissue sampling. Blood was collected by cardiac puncture, and the livers were isolated, immediately freeze-clamped in liquid nitrogen and stored at $-80^{\circ} \mathrm{C}$ until analysis. Serum levels of alanine aminotransferase (ALT) and TG were assayed using an ABX Pentra400 clinical chemistry analyser. TG content was extracted from frozen liver tissues and determined as previously described [20].

For the histological examination, livers from killed mice were immediately incised and weighed. Small pieces from each lobe were fixed in 10\% formalin and stained with haematoxylin and eosin and ORO for histological analysis. The histological features were grouped into five broad categories: steatosis, inflammation, hepatocellular injury, fibrosis and miscellaneous features. The system of evaluation was adapted from the original proposed by Kleiner et al [24] for the histological evaluation of NASH and took into account the following histological variables: steatosis grade (quantified by low- to medium-power evaluation of parenchymal involvement by steatosis): score $0,<5 \%$; score $1,5-33 \%$; score 2 , $>33-66 \%$, score 3,>66\%; location (predominant distribution pattern): zone 3 , score 0 ; zone 1 , score 1 ; azonal, score 2 ; inflammation: lobular inflammation (overall assessment of all inflammatory foci): score 0 , no foci; score $1,<2$ foci per $\times 200$ field; score 2, 2-4 foci per $\times 200$ field; score $3,>4$ foci per $\times 200$ field; necrosis: score 0 , present; score 1 , absent.

Cell culture procedures and transfection Human hepatocellular carcinoma (HepG2) cells and NMuLi mouse liver cells were cultured at $37^{\circ} \mathrm{C}$ in DMEM supplemented with 10\% FBS, 2\% L-glutamine, 10,000 units/ml penicillin and 10,000 $\mu \mathrm{g} / \mathrm{ml}$ streptomycin. Transient transfection of Prep1, Prep $1_{H R 1}$ and Pbx1 plasmids or Ship2 (5'-GCTCTCTAGTTCCTGCTCCC-3') phosphorothioate antisense oligonucleotides or Ship2 (5'-TCGCCACGTCGCTCATTGTT-3') control scrambled oligonucleotides were performed by the Lipofectamine method according to the manufacturer's instruction (Invitrogen). PREP1 stable clones were selected as previously described [20].

ORO staining To measure cellular neutral lipid droplet accumulation, HepG 2 cells were stained by the ORO method as previously described [25].

Western blot analysis Tissue samples were homogenised in a Polytron Homogenizer (Brinkmann Instruments, NY, USA) in $20 \mathrm{ml}$ T-PER reagent ( $\mathrm{g}$ tissue $)^{-1}$ according to the manufacturer's instructions (Pierce, IL, USA). Total cell lysates were obtained and separated by SDS-PAGE as previously described [26].

Real-time RT-PCR analysis Total cellular RNA was isolated from liver tissue and HepG2 cells using the RNeasy Kit (Qiagen Sciences, Germantown, MD, USA), according to the manufacturer's instructions.

A total of $1 \mu \mathrm{g}$ of tissue or cell RNA was reversetranscribed using SuperScript II Reverse Transcriptase (Invitrogen). PCR reactions were analysed using SYBR Green mix (Bio-Rad, Hercules, CA, USA). Reactions were performed using the Platinum SYBR Green qPCR SuperMix-UDG using an iCycler IQ Multi-Color Real Time PCR Detection System (Bio-Rad). All reactions were performed in triplicate and $\beta$-actin was used as an internal standard. The primer sequences used are described in electronic supplementary material (ESM) Table 1.

Chromatin immunoprecipitation and luciferase assays Chromatin immunoprecipitation (ChIP) and re-ChIP assays were performed using NMuLi cells as previously described [27]. For luciferase assays, the mouse Ship2 fragment $-1,385$ bp to $-1,115$ bp was amplified by PCR from genomic mouse DNA isolated from the murine liver cell line $(\mathrm{NMuLi}$ cells). The following primers were used: forward: 5'-KpnICAAAGGGGAAGCTGGAAACGGGA-3' and reverse: 5'-XhoI-TTAGCCGAAGGCTAGAGGGTGCT-3', where KpnI and XhoI indicate the restriction sites added to the sequence. The amplified fragments were cloned in the pGL3 
promoter vector (Promega, Madison, WI, USA) by KpnI and XhoI. NMuLi cells were cotransfected with $2 \mu \mathrm{g}$ of Ship2 promoter vector together with different amounts of Prep1 and $P b x 1$ expression vectors. Total DNA content (up to $4 \mu \mathrm{g} /$ plate) was normalised to the empty vector devoid of Prep 1 and Pbxl coding sequence. Forty-eight hours after transfection the cells were harvested and lysed as described previously [27]. Luciferase activity was measured by a commercial luciferase assay kit (Promega). Values were normalised for beta-galactosidase.

Statistical procedures Data were analysed with StatView software (Abacus Concepts, Piscataway, NJ, USA) by two-factor ANOVA. $p$ values of $<0.05$ were considered statistically significant [28].

\section{Results}

Prep1 impact on lipogenesis-controlling signalling events in mouse liver Serum TG content, but not hepatic ApoB100 (also known as $A p o b$ ) expression or serum VLDL secretion, was reduced by $33 \%$ in Prep ${ }^{i /+}$ mice compared with their nonhypomorphic littermates (WT) (Fig. 1a-c). Fas expression
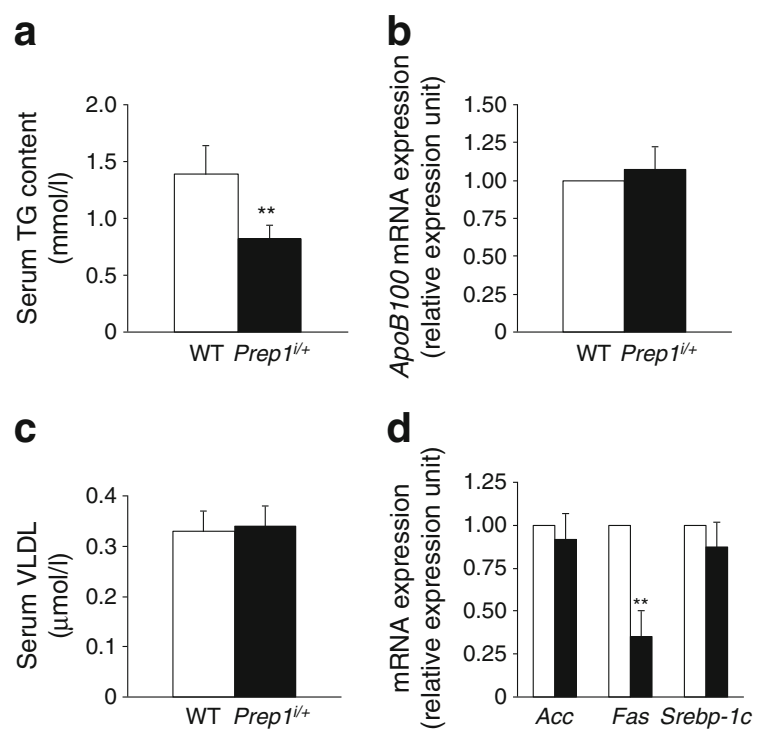

Fig. 1 Serum TG and VLDL content and Fas mRNA levels in Prep1 ${ }^{i /+}$ mice. (a) Serum TG content was measured as described in the Methods. Each bar represents the mean $\pm \mathrm{SD}$ of three independent experiments in triplicate (eight mice/genotype). (b) Hepatic levels of ApoB100 mRNA were quantified by real-time RT-PCR analysis, using $\beta$-actin as the internal standard. (c) Serum VLDL was measured by ELISA assay, as described in the Methods. Each bar represents the mean \pm SD of three independent experiments in triplicate (eight mice/genotype). (d) The abundance of Acc, Fas and Srebp-1c mRNA was determined by realtime RT-PCR analysis of total RNA isolated from the liver of Prep1 $1^{i /+}$ (black bars) and control mice (white bars), using $\beta$-actin as the internal standard. (b, d) Bars represent the mean \pm SD of four independent experiments, in each of which the reactions were performed in triplicate using the pooled total RNAs obtained from six mice/genotype. ${ }^{* *} p<0.01$ decreased by $65 \%$ in Prep $^{i /+}$ compared with WT mice, while $A c c$ and Srebp-1c (encoding sterol regulatory element binding protein-1c) were not significantly affected in Prep $1^{i /+}$ mice (Fig. 1d). To investigate this issue in greater detail, we first focused our attention on one of the major lipogenesis regulatory enzymes, AMPK, and on its downstream target, ACC. Active AMPK, phosphorylated at Thr172, was significantly upregulated in Prep $1^{i / 4}$ mice, with no change in AMPK protein levels. Indeed, the pAMPK to AMPK ratio was threefold higher in Prepl ${ }^{i /+}$ compared with WT mice. The expression and phosphorylation of ACC at Ser79 closely matched those of AMPK (Fig. 2a). We next examined the upstream signalling mechanisms converging on AMPK and assayed phosphorylation of both LKB1 and CAMKK $\beta$. As shown in Fig. $2 b$, LKB1 exhibited almost threefold increased phosphorylation in Prep $^{i / 4}$ mice, while CAMKK $\beta$ exhibited no difference between Prep $^{i /+}$ and WT mice.

Several protein kinases play a major role in transducing regulatory signals to LKB1 $[29,30]$. In particular, atypical $\mathrm{PKC} \zeta$ and PKA have been reported to represent major LKB1 regulators. In our Prep1 ${ }^{i /+}$ mice only $\mathrm{PKC} \zeta$ exhibited

\section{a}
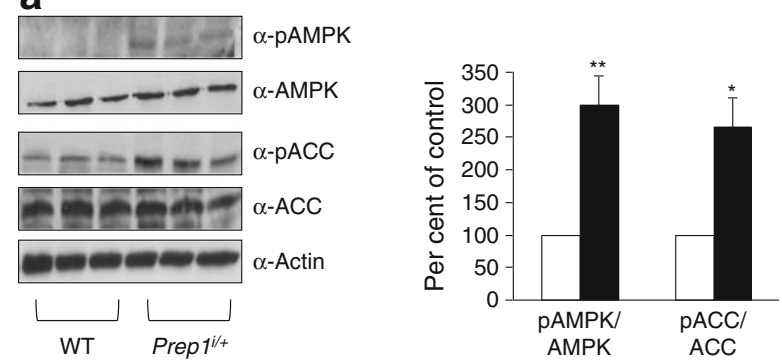

b
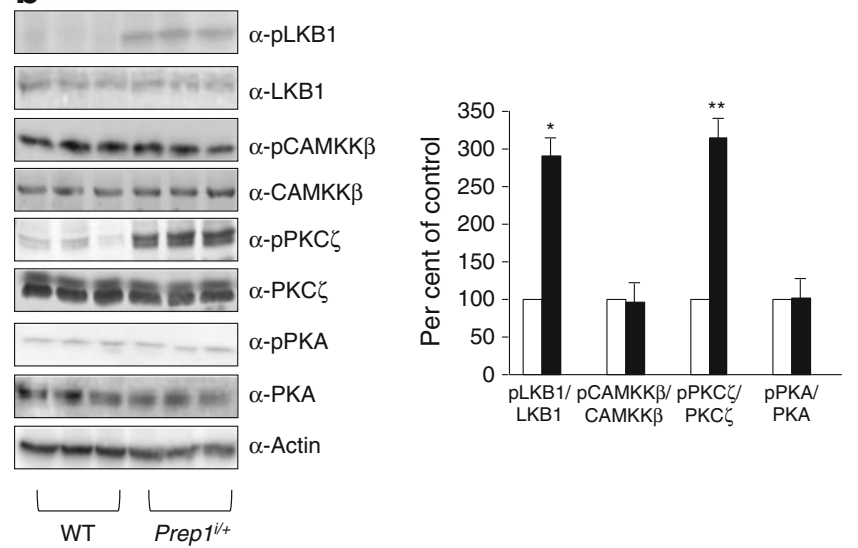

Fig. 2 AMPK signalling in Prep $1^{i /+}$ mice. (a) Liver tissue from Prep $^{i /+}$ (black bars) and control mice (white bars) was dissected and solubilised, and protein samples were analysed by western blot with pAMPK, AMPK, pACC and ACC antibodies. (b) Aliquots of the lysates were also blotted with $\mathrm{pLKB} 1, \mathrm{LKB} 1, \mathrm{pCAMKK} \beta, \mathrm{CAMKK} \beta, \mathrm{pPKC} \zeta$, $\mathrm{PKC} \zeta$, pPKA and PKA antibodies. Actin antibody was used for normalisation. The blots shown are representative of four independent experiments. Bars represent the mean $\pm \mathrm{SD}$ of densitometric values from the four experiments. ${ }^{*} p<0.05, * * p<0.01$ 
enhanced phosphorylation, as phosphorylation of PKA was very comparable in Prep ${ }^{i /+}$ and WT mice (Fig. 2b).

To further explore the impact of PREP1 on hepatic lipogenesis, we transiently transfected Prep1 cDNA in HepG2 cells (Fig. 3a). We then analysed the lipid accumulation in these cells by ORO staining. As shown in Fig. 3b, ORO staining was twofold higher in Prepl-overexpressing cells than in controls transfected with an empty vector. Fas, but not Acc, mRNA levels also increased by $50 \%$ in the Prepltransfected cells (Fig. 3c), while phosphorylation of $\mathrm{PKC} \zeta$, LKB1, AMPK and ACC was significantly reduced (Fig. 3d).

Prep1 regulates hepatic lipogenesis via Ship2 PREP1 regulation of hepatic gluconeogenesis occurs through a PREP1/ PBX1 complex [20]. We therefore hypothesised that PBX1 co-regulates the PREP1 action on lipogenesis. To examine this possibility, we transfected HepG2 cells with either Pbxl or Prep $1_{H R 1}$ cDNA. The latter is unable to bind and form a complex with PBX1 [21]. Similarly to PREP1, PBX1 was found to downregulate the PKC C/LKB1/AMPK/ACC phosphorylation cascade and induce Fas expression and intracellular lipid droplet accumulation in the liver cells. In contrast, Prep $1_{\text {HRI }}$ transfection elicited no effect on these mechanisms, indicating that PBX1 binding is necessary for PREP1 control of lipogenesis (ESM Fig. 1).

Published evidence indicates that the lipid phosphatase SHIP2 reduces cellular levels of phosphatidylinositol-3-kinasegenerated phosphatidylinositol-3-phosphate and $\mathrm{PKC} \zeta$ activation [31-33]. Interestingly, SHIP2 protein and mRNA levels in the livers of Prepl $1^{i /+}$ mice were reduced by more than $60 \%$ compared with WT (Fig. 4a, b). Transfection of either Prepl or Pbxl cDNAs in HepG2 cells induced a close to twofold increase in SHIP2 protein and mRNA expression, while transfection of the Prep $1_{H R I}$ mutant caused no change (Fig. 4c, d). We therefore used Ship2-specific antisense oligonucleotides to test the hypothesis that SHIP2 represents a downstream mediator of the PREP1/PBX1 action on hepatic lipogenesis. Indeed, transfection of Ship2-specific antisense oligonucleotides in HepG2 $2_{\text {PREP1c }}$ cells (which overexpress Prep1 by fivefold) [20] caused a 75\% reduction in SHIP2 levels and, in parallel, generated a phenotype similar to that observed in Prep $1^{i /+}$ mice, with a more than fourfold increase in AMPK and ACC phosphorylation and downregulated Fas, but not $A c c$, expression (Fig. 5). PKCC and LKB1 phosphorylation was also significantly induced in Ship2-specific antisense oligonucleotide transfected cells (data not shown).

Prep1 regulation of Ship2 gene transcription Bioinformatic analysis revealed the presence of a potential PREP1/PBX1 binding site at position $-1,284$ to $-1,268$ upstream of the transcription initiation site of the Ship2 gene (Fig. 6a). To validate this putative region, we used specific antibodies against either PREP1 or PBX1 and performed ChIP and reChIP studies in NMuLi mouse liver cells. These experiments revealed the simultaneous presence of both Prep 1 and Pbx1 at the putative binding site (Fig. 6b). We than cloned the PREP1/ PBX1 binding fragment into a pGL3 promoter construct upstream of the luciferase gene. This construct was then cotransfected into NMuLi cells together with Prep1, Pbx1, both the transcription factors and Prep $1_{H R l}$ cDNAs, followed
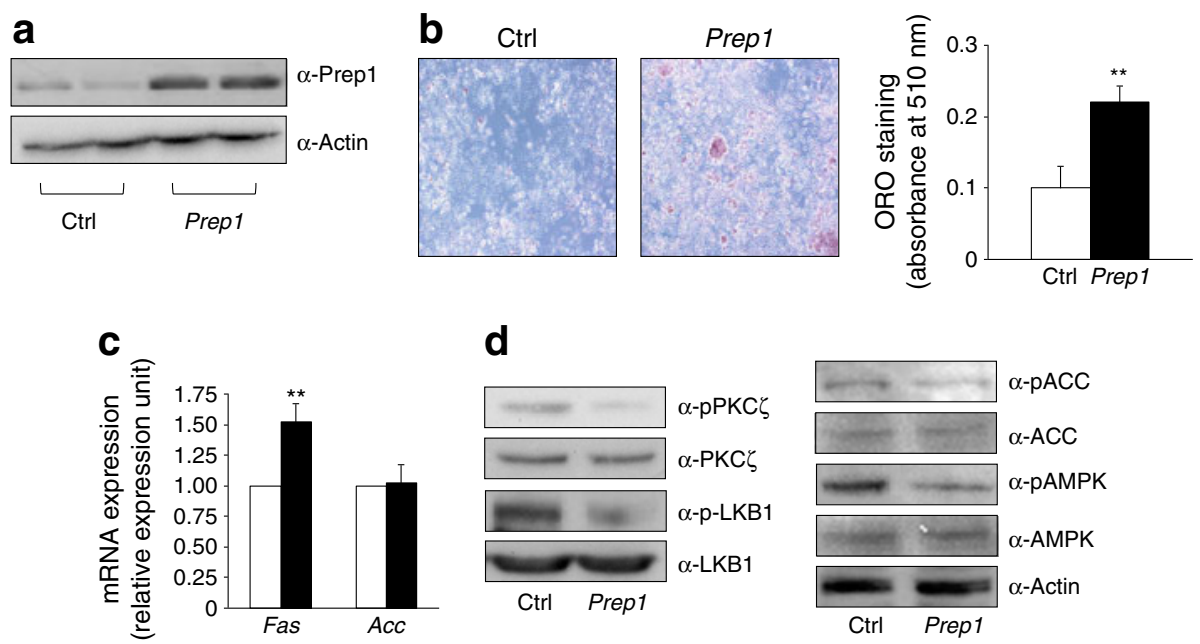

d
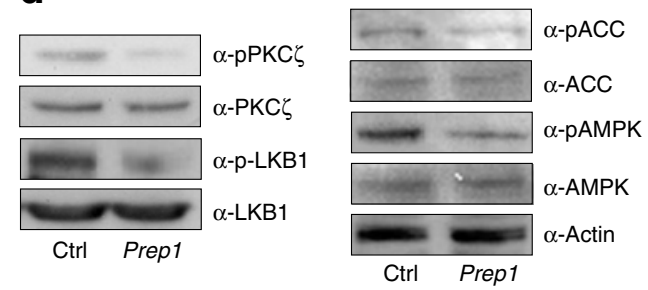

Fas and Acc mRNA in cells transfected with Prep 1 cDNA (black bars) or in control cells transfected with an empty vector (white bars) were quantified by real-time RT-PCR analysis, using $\beta$-actin as the internal standard. Bars represent the mean $\pm \mathrm{SD}$ of four independent experiments. $* * p<0.01$. (d) HepG2 cell lysates were western blotted with $\mathrm{pPKC} \zeta$, PKC $\zeta$, pLKB1, LKB1, pACC, ACC, pAMPK or AMPK antibodies, as outlined in the legend to Fig. 2. The blots shown are representative of four independent experiments. Ctrl, control 


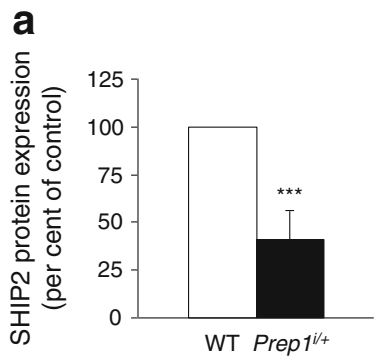

b

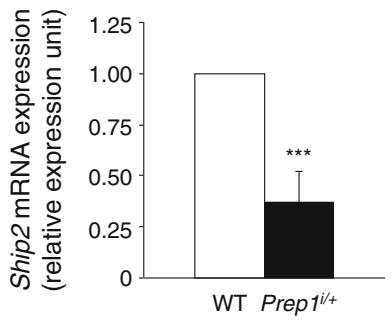

C

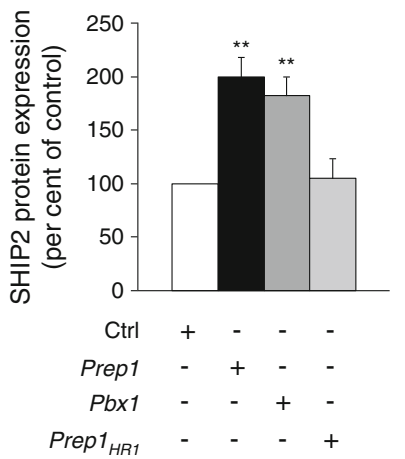

d

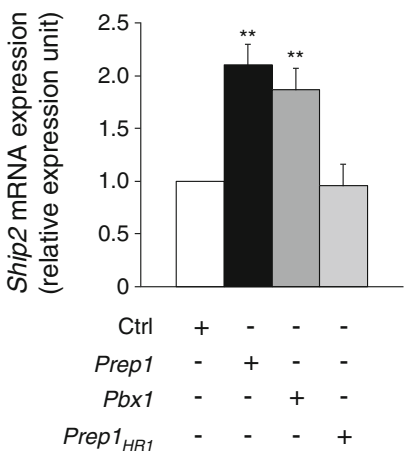

Fig. 4 Ship2 expression in Prep $1^{i / 4}$ mice and in HepG2-overexpressing PREP1. (a) SHIP2 protein abundance was analysed by western blotting lysates of hepatic tissue from Prep $1^{i /+}$ and control mice using specific antibodies. Actin antibodies were used for normalisation. Each bar represents the mean $\pm \mathrm{SD}$ of duplicate determinations in ten mice/group. (b) The abundance of Ship 2 mRNA was determined by real-time RT-PCR analysis of total RNA isolated from the liver of Prep ${ }^{i /+}$ and control mice, using $\beta$-actin as the internal standard. Bars represent the mean \pm SD of five independent experiments, each of which were performed in triplicate using the pooled total RNAs obtained from six mice/genotype. (c) Lysates from control or overexpressing Prep1, Pbxl and Prep1 ${ }_{H R I}$ cDNAs HepG2 cells were blotted with SHIP2 antibodies. Actin antibodies were used for normalisation. Each bar represents the mean $\pm \mathrm{SD}$ of duplicate determinations from four independent experiments. (d) The levels of Ship 2 mRNA in cells transfected as described above were quantified by real-time RT-PCR analysis, using $\beta$-actin as the internal standard. Each bar represents the mean $\pm \mathrm{SD}$ of duplicate determinations from four independent experiments. $*^{* *} p<0.01, * * * p<0.001$. Ctrl, control

by measurement of luciferase activity. As shown in Fig. 6c, Prepl and Pbxl enhanced luciferase activity by 17 -fold, while Prep $1_{H R I}$ did not elicit any effect.

Prep $^{i / 4}$ mice are resistant to diet-induced steatohepatitis To assess the in vivo significance of PREP1 control of hepatic lipogenesis, we analysed the effect of a steatogenic MCDD in Prep $^{i /+}$ mice. After 4 weeks of the MCDD, both Prep $1^{i /+}$ mice and their non-hypomorphic littermates showed significant weight loss (Table 1), which was independent of food intake (data not shown). Liver weight also comparably decreased in both of the mice groups upon diet administration. Both serum ALT and TG levels were lower in the Prep1 $1^{i / 4}$ mice at baseline. Interestingly, the increase in ALT levels and decrease in TG levels following the regimen was significantly less pronounced in Prep1 ${ }^{i / 4}$ compared with control mice. In addition, MCDD-induced accumulation of TGs was 25\% reduced in Prep1 ${ }^{i / 4}$ mouse liver (Fig. 7a). Indeed, livers from MCDD-fed WT mice revealed very significant morphological evidence of steatosis (score 2-3) accompanied by severe lobular inflammation (score 2-3) and foci of necrosis (Fig. 7b) as indicated by the arrows. In comparison, Prep $1^{i /+}$ mice showed only grade 1 hepatic steatosis, grade $0-1$ inflammation and absence of necrosis. In addition, little ORO staining accumulation occurred in these mice in comparison with their non-hypomorphic littermates. When fed a regular chow diet, these animals showed no histological differences in livers, suggesting that PREP1 expression levels determine the hepatocyte sensitivity to a steatogenic diet.

\section{Discussion}

NAFLD spans from steatosis to NASH, with lobular inflammatory and necrotic lesions and fibrosis [34]. Metabolic abnormalities, including dysregulated insulin signalling and action, and nutritional and genetic factors are well-established contributors to the onset and progression of these liver disorders [7]. Indeed, patients with insulin resistance syndrome have a four- to eleven-fold increased risk of developing NAFLD [9]. In addition, small-interfering-RNA-mediated suppression of IRS1 and IRS2 results in hepatic insulin resistance, glucose intolerance and hepatic steatosis in rodents $[35,36]$. However, the identity of the genes affecting the risk of NAFLD and of NASH as well as the molecular mechanisms involved in their function have been only partially uncovered. a

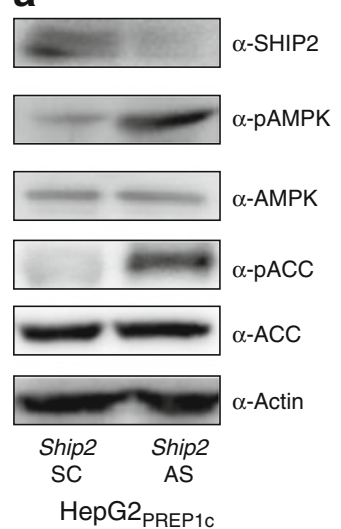

b

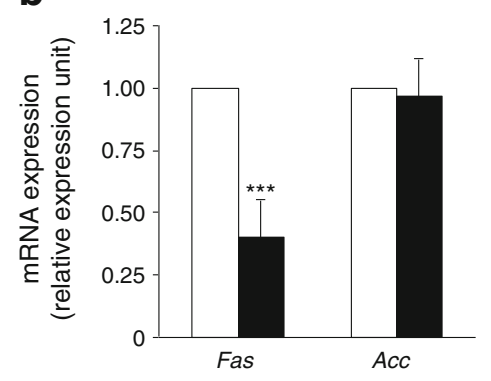

Fig. 5 Effect of Ship2 antisense on lipogenesis regulation in PREP1transfected HepG2 cells. (a) HepG2 $2_{\text {PREP1c }}$ cells were transfected with specific Ship2 phosphorothioate antisense oligonucleotides (Ship2 AS) or scrambled oligonucleotides (Ship2 SC) and analysed by western blot with SHIP2, pAMPK, AMPK, pACC, ACC or actin antibodies. The blots shown are representative of four independent experiments. (b) The levels of Fas and Acc mRNA in HepG2 $2_{\text {PREP1c }}$ transfected with Ship2 AS (black bars) or a Ship2 SC (white bars) were quantified by real-time RT-PCR analysis, using $\beta$-actin as the internal standard. Bars represent the mean $\pm \mathrm{SD}$ of four independent experiments. $* * * p<0.001$ 
a 4,000 bp upstream Ship2 gene

...ATATAGATTGACTGGGT..
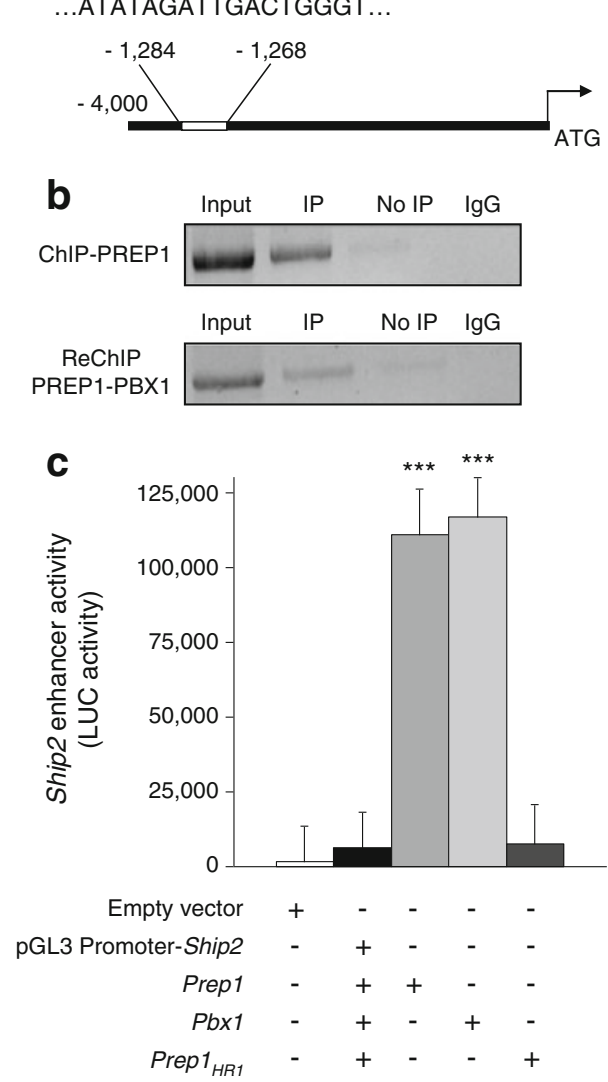

Fig. 6 PREP1/PBX1 regulation of Ship2 reporter gene activity. (a) Schematic representation of the $5^{\prime}$ sequence upstream of the putative Ship2 transcription start site with the potential binding site for PREP1 and PBX1. (b) Soluble chromatin was prepared from NMuLi mouse liver cells and immunoprecipitated with PREP1 antibodies. Total (input) and immunoprecipitated DNAs were amplified using primer pairs encompassing the indicated Ship2 fragments. A re-ChIP assay with PBX1 antibodies was performed as described in the Methods. (c) NMuLi cells were transfected with $2 \mu \mathrm{g}$ of the Ship 2 enhancer-luciferase construct alone or in combination with Prep 1, Pbxl or the mutant Prep $_{H R I}$. Luciferase (LUC) activity was assayed and normalised as described in the Methods. Bars represent mean values $\pm \mathrm{SD}$ of determinations from four independent experiments. $* * * p<0.001$

Previous work in our laboratory revealed that PREP1 attenuates insulin glucoregulatory function in liver by transcriptional activation of the Shp1 (also known as Ptpn6) gene, a known silencer of insulin signalling [20]. In addition, we showed that Prep1 ${ }^{i / 4}$ mice feature reduced hepatic TG content. In the present work, we demonstrate that these mice also exhibit depressed serum TG levels. This effect is not due to changes in VLDL secretion or in hepatic ApoB100 expression, which are required for TG transport in plasma [37]. However, we found that the reduced serum TG level featured by the Prep $1^{i /+}$ mice was accompanied by reduced Fas expression, prompting us to explore the hypothesis that PREP1 also controls hepatic lipid synthesis. Indeed, we have shown that, in livers from Prep $^{i /+}$ mice, the major inhibitor of de novo lipogenesis, AMPK, was strongly induced, accompanied by an increase in ACC phosphorylation. AMPK regulates hepatic lipid metabolism by exerting short- and longterm effects. Short-term regulation includes AMPK phosphorylation and inactivation of ACC, thereby inhibiting fatty acid biosynthesis. Long-term effects include AMPK regulation of lipogenesis by inhibition of SREBP-1c and promotion of lipogenic gene expression, such as Acc and Fas [3]. Because Srebp-1c and Acc expression were not reduced in Prep $1^{i /+}$ mice, the short-term effects of AMPK might have prevailed in these Prep $^{i / 4}$ mice. Indeed, PKC $/ / L K B 1$ signalling was also induced in Prepl-deficient mouse liver. Work by Luna and co-workers has demonstrated that metformin-induced activation of AMPK is accompanied by induction of PKC $\zeta$ [38]. Importantly, genetic ablation as well as pharmacologic inhibition of PKC $\zeta$ prevent stimulation of both LKB1 and AMPK [29], indicating that PREP1 lipogenic signalling upstream of AMPK involves PKC $\zeta$ upregulation. Consistent with this mechanism, we show that Prepl overexpression in liver cells downregulates $\mathrm{PKC} \zeta$ function and AMPK activity and enhances lipid accumulation. Previous work in our laboratory demonstrated that Prep1 silencing enhances liver insulin sensitivity and insulin-dependent glycogen synthesis [20]. In contrast, lipogenesis was downregulated in the Prep $1^{i /+}$ mice. Downregulation of lipogenesis might be independent of and prevailing on the enhanced insulin sensitivity caused by Prep1 silencing. In addition, the increased liver sensitivity to insulin might be confined to glycogen synthesis and not present in other pathways.

In vivo, genetic ablation of the inositol phosphate phosphatase SHIP2 determines a phenotype reminiscent of that of
Table 1 Characterisation of WT and Prep $1^{i /+}$ mice

Values are means $\pm \mathrm{SD}$

${ }^{*} p<0.05 ; * * p<0.01 ; * * * p<0.001$ for differences between animals fed an MCDD vs control diet

${ }^{\dagger} p<0.05 ;{ }^{\dagger} p<0.01$ for differences between Prepl $^{i+4}$ vs WT mice

\begin{tabular}{|c|c|c|c|c|}
\hline \multirow[t]{2}{*}{ Variable } & \multicolumn{2}{|l|}{ Control diet } & \multicolumn{2}{|l|}{ MCDD } \\
\hline & WT & Prep $1^{i /+}$ & WT & Prep1 ${ }^{i /+}$ \\
\hline Initial body weight (g) & $28.7 \pm 1.0$ & $25.5 \pm 0.6$ & $28 \pm 0.12$ & $27.2 \pm 0.5$ \\
\hline Final body weight (g) & $30.4 \pm 0.9$ & $30.1 \pm 0.4$ & $19.1 \pm 0.7 *$ & $18.8 \pm 1.0^{* * *}$ \\
\hline Liver weight (g) & $1.16 \pm 0.9$ & $1.06 \pm 0.5$ & $0.86 \pm 0.5 *$ & $0.81 \pm 0.4^{*}$ \\
\hline Serum ALT level ( $\mu$ kat/l) & $1.47 \pm 0.11$ & $1.09 \pm 0.1$ & $5.13 \pm 0.1 * *$ & $2.59 \pm 0.01 * * \dagger \dagger$ \\
\hline Serum TG level (mmol/l) & $1.18 \pm 0.05$ & $0.85 \pm 0.03^{\dagger}$ & $0.87 \pm 0.04 *$ & $0.68 \pm 0.05^{* \dagger}$ \\
\hline
\end{tabular}



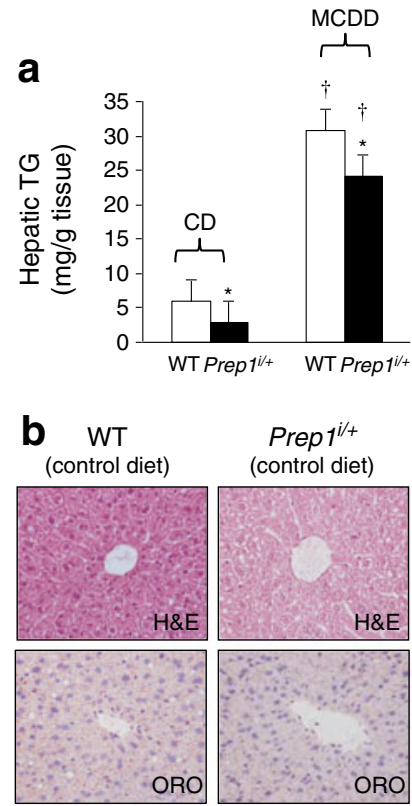

Prep $^{i / 4}$
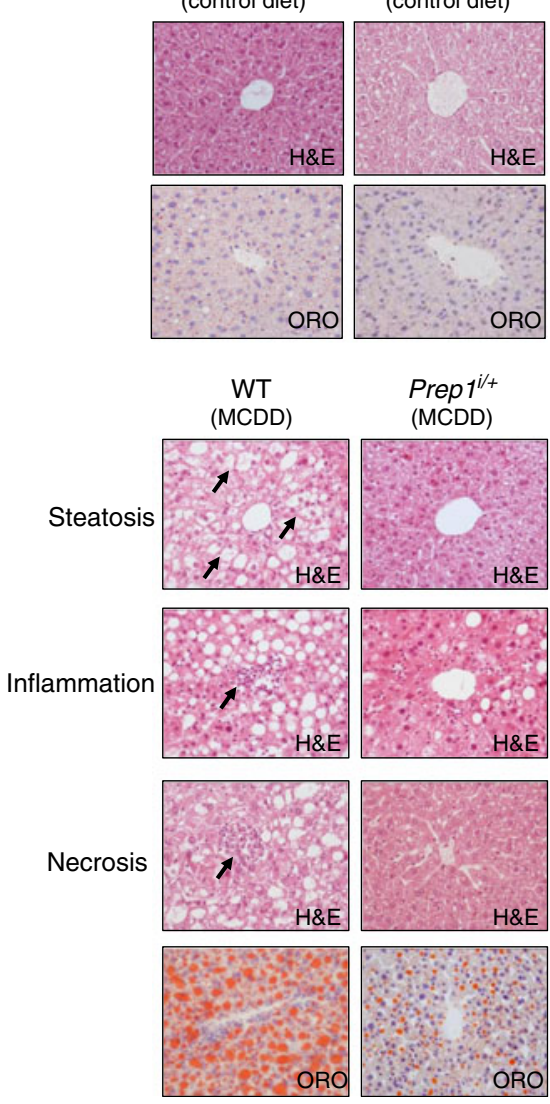

Fig. 7 Effect of the MCDD on Prep1 $1^{i / 4}$ mice. (a) Prep1 $1^{i / 4}$ and control mice (nine mice/group) were fed an MCDD or control diet (CD) and intrahepatic TG content was measured as described in the Methods. Bars represent the means $\pm \mathrm{SD}$ of values obtained from each group of animals. ${ }^{*} p<0.05$, MCDD vs CD ${ }^{\dagger} p<0.05$, Prep $^{i /+}$ vs WT mice. (b) Representative liver histology of WT and Prep1 ${ }^{i / 4}$ mice fed a control diet and MCDD. Liver sections were stained with haematoxylin and eosin (H\&E) or ORO and examined by light microscopy with a magnification of $\times 40$; arrows show foci of steatosis, inflammation and necrosis

Prep $1^{i /+}$ mice. First, both Ship2 -null mice and Prep ${ }^{i /+}$ mice feature enhanced peripheral sensitivity to insulin [39]. Second, serum TG levels are significantly reduced in both of these genetic models [39], raising the possibility that, at least in part, the Prep $1^{i / 4}$ mouse phenotype is determined by reduced Ship2 expression and/or function. This proved to be correct, as Ship 2 mRNA as well as protein levels were found to be strongly reduced in the livers of Prep $1^{i /+}$ mice, while the opposite changes occurred in liver cells transfected with Prep1 cDNA. Of importance, antisense silencing of Ship2 in hepatocytes prevents signalling activation through the PKC C/LKB1/AMPK cascade and Fas induction caused by PREP1 overexpression. These findings indicate that the Ship2 gene represents a transcriptional target of PREP1, upstream of the AMPK control of hepatic lipogenesis. The SHP1 and SHP2 tyrosine phosphatases are also upregulated by PREP1 [20]. Both of these phosphatases may silence PKC $\zeta$ by attenuating insulin signalling in cells expressing increased levels of Prep1. In addition, recent work by Nagata and co-workers revealed that liver-specific ablation of SHP2 protects mice from developing liver steatosis [40]. However, antisense silencing of SHP1, SHP2 or both of these phosphatases in liver cells has negligible effects on the increased TG synthesis caused by PREP1 overexpression (data not shown), indicating a marginal, if any, role in PREP1 control of liver lipogenesis.

The mechanistic details involved in PREP1 control of SHIP2 function and the hypothesis that they may include direct transcriptional regulation are addressed in the present paper. We first identified a putative PREP1 binding site in the Ship2 regulatory region upstream of the transcription initiation site of the gene. Subsequent ChIP experiments demonstrated PREP1 binding to this putative site. In addition, we have shown that this binding enhances Ship2 reporter activity in luciferase assays. These results are also supported by the analysis of ChIPsequencing experiments performed on human A549 cells overexpressing PREP1, which identified a weak PREP1 binding site about $1.3 \mathrm{~kb}$ upstream of the Ship 2 transcription start site. Moreover, in NIH 3T3-L1 pre-adipocytes, the downregulation of Prep1 induces the loss of the H3K4Me1 chromatin mark, which is specific for enhancers; this effect is not generalised to other genes (data not shown).

Further ChIP/re-ChIP experiments revealed the simultaneous presence of the major PREP1 co-regulatory factor PBX1 [15] at the Ship2 promoter. We showed that PBX1 is required for PREP1 regulation of lipogenesis since: (1) PBX1 mimics PREP1 signalling through the SHIP2/PKC C/LKB1/ AMPK/ACC cascade; and (2) expression of the PREP1 $1_{\mathrm{HR} 1}$ mutant, which is unable to bind PBX1, prevents PBX1 recruitment at the Ship2 promoter and, simultaneously, prevents the induction of lipogenic signalling. Previous studies in liver have demonstrated that PREP1 controls insulin glucoregulatory function by transcriptional targeting of the $S h p 1$ tyrosine phosphatase [20]. As in the case of lipogenesis, overexpression of PBX1 mimics PREP1 action on glucose homeostasis. Thus, in the liver, transcriptional targeting of both lipid and tyrosine phosphatases occurs in partnership with PBX1.

An MCDD results in liver injury similar to human NASH [41]. In addition to increased fatty acid uptake and decreased VLDL secretion, enhanced lipogenesis contributes to the hepatic accumulation of TGs in livers of MCDD-treated mice [42]. Similar mechanisms may predispose to hepatocyte 
damage in human NASH [43]. However, the molecular and genetic mechanisms responsible for NASH have been only partially uncovered. In the present report, we show that silencing of the Prep1 gene protects mice from the TG accumulation and hepatocyte damage caused by MCDD administration, indicating that PREP1 may represent a molecular link between insulin resistance and the development of hepatic steatosis. Whether PREP1 itself or nearby loci affect lipid metabolism or are implicated in NAFLD in humans remains to be clarified. Current evidence indicates that PREP1 expression is increased in patients with alcoholic steatohepatitis, supporting the hypothesis that PREP1 may also have a role in humans [44].

Funding This work was supported by the European Foundation for the Study of Diabetes, the European Commission's PREPOBEDIA project (201681) and the Ministero dell'Università e della Ricerca Scientifica (PRIN and FIRB: RBIP0689BS). The financial support of Telethon-Italy to $\mathrm{FBe}$ and $\mathrm{FBl}$ is also gratefully acknowledged.

Duality of interest The authors declare that there is no duality of interest associated with this manuscript.

Contribution statement $\mathrm{FO}$ and SC were the main contributors in terms of conception, design, acquisition and interpretation of data and drafting the article. AL, ML, LP, TBP, GAR, DP, OP and CM contributed to conceptual design and the acquisition of data. $\mathrm{PF}$ and $\mathrm{FBl}$ contributed to conceptual design, analysis and interpretation of data and discussion of the results. FBe contributed to conceptual design, interpretation and discussion of the results and supervision of the overall work. All of the authors critically revised the article and approved the final version.

\section{References}

1. Postic C, Dentin R, Girard J (2004) Role of the liver in the control of carbohydrate and lipid homeostasis. Diabetes Metab 30:398-408

2. Witters LA, Kemp BE (1992) Insulin activation of acetyl-CoA carboxylase accompanied by inhibition of the 5'-AMP-activated protein kinase. J Biol Chem 267:2864-2867

3. Viollet B, Foretz M, Guigas B et al (2006) Activation of AMPactivated protein kinase in the liver: a new strategy for the management of metabolic hepatic disorders. J Physiol 574:41-53

4. Towler MC, Hardie DG (2007) AMP-activated protein kinase in metabolic control and insulin signaling. Circ Res 100:328-341

5. Carling D (2004) The AMP-activated protein kinase cascade-a unifying system for energy control. Trends Biochem Sci 29:18-24

6. Shackelford DB, Shaw RJ (2009) The LKB1-AMPK pathway: metabolism and growth control in tumour suppression. Nat Rev Cancer 9:563-575

7. Di Rosa M, Malaguarnera L (2012) Genetic variants in candidate genes influencing NAFLD progression. J Mol Med 90:105-118

8. Dongiovanni P, Anstee QM, Valenti L (2013) Genetic predisposition in NAFLD and NASH: impact on severity of liver disease and response to treatment. Curr Pharm Des 19:5219-5238

9. Nagle CA, Klett EL, Coleman RA (2009) Hepatic triacylglycerol accumulation and insulin resistance. J Lipid Res 50(Suppl):S74-S79

10. Struben VM, Hespenheide EE, Caldwell SH (2000) Nonalcoholic steatohepatitis and cryptogenic cirrhosis within kindreds. Am J Med 108:9-13

11. Willner IR, Waters B, Patil SR, Reuben A, Morelli J, Riely CA (2001) Ninety patients with nonalcoholic steatohepatitis: insulin resistance, familial tendency, and severity of disease. Am J Gastroenterol 96: 2957-2961

12. Anstee QM, Daly AK, Day CP (2011) Genetic modifiers of nonalcoholic fatty liver disease progression. Biochim Biophys Acta 1812:1557-1566

13. Guichelaar M, Gawrieh S, Olivier M et al (2013) Interactions of allelic variance of PNPLA3 with non genetic factors in predicting NASH and non-hepatic complications of severe obesity. Obesity. doi: 10.1002/oby.20327

14. Al-Serri A, Anstee QM, Valenti L et al (2012) The SOD2 C47T polymorphism influences NAFLD fibrosis severity: evidence from case-control and intra-familial allele association studies. J Hepatol $56: 448-454$

15. Berthelsen J, Zappavigna V, Ferretti E, Mavilio F, Blasi F (1998) The novel homeoprotein Prep1 modulates $\mathrm{Pbx}-\mathrm{Hox}$ protein cooperativity. EMBO J 17:1434-1445

16. Berthelsen J, Kilstrup-Nielsen C, Blasi F, Mavilio F, Zappavigna V (1999) The subcellular localization of PBX1 and EXD proteins depends on nuclear import and export signals and is modulated by association with PREP1 and HTH. Genes Dev 13:946-953

17. Berthelsen J, Zappavigna V, Mavilio F, Blasi F (1998) Prep1, a novel functional partner of $\mathrm{Pbx}$ proteins. EMBO J 17:1423-1433

18. Penkov D, Mateos San Martin D, Fernandez-Diaz LC et al (2013) Analysis of the DNA-binding profile and function of TALE homeoproteins reveals their specialization and specific interactions with Hox genes/proteins. Cell Rep 3:1321-1333

19. Oriente F, Fernandez Diaz LC, Miele C et al (2008) Prep1 deficiency induces protection from diabetes and increased insulin sensitivity through a p160-mediated mechanism. Mol Cell Biol 28:5634-5645

20. Oriente F, Iovino S, Cabaro S et al (2011) Prep1 controls insulin glucoregulatory function in liver by transcriptional targeting of SHP1 tyrosine phosphatase. Diabetes 60:138-147

21. Diaz VM, Mori S, Longobardi E et al (2007) p160 Myb-binding protein interacts with Prep1 and inhibits its transcriptional activity. Mol Cell Biol 27:7981-7990

22. Penkov D, Di Rosa P, Fernandez Diaz L et al (2005) Involvement of Prep1 in the alphabeta T cell receptor T-lymphocytic potential of hematopoietic precursors. Mol Cell Biol 25:10768-10781

23. Ferretti E, Villaescusa JC, Di Rosa P et al (2006) Hypomorphic mutation of the TALE gene Prep1 (pKnox1) causes a major reduction of $\mathrm{Pbx}$ and Meis proteins and a pleiotropic embryonic phenotype. Mol Cell Biol 26:5650-5662

24. Kleiner DE, Brunt EM, van Natta M et al (2005) Design and validation of a histological scoring system for nonalcoholic fatty liver disease. Hepatology 41:1313-1321

25. Ramirez-Zacarias JL, Castro-Munozledo F, Kuri-Harcuch W (1992) Quantitation of adipose conversion and triglycerides by staining intracytoplasmic lipids with Oil Red O. Histochemistry 97:493-497

26. Miele C, Caruso M, Calleja V et al (1999) Differential role of insulin receptor substrate (IRS)-1 and IRS-2 in L6 skeletal muscle cells expressing the Arg1152 --> Gln insulin receptor. J Biol Chem 274: 3094-3102

27. Ungaro P, Teperino R, Mirra P et al (2008) Molecular cloning and characterization of the human PED/PEA-15 gene promoter reveal antagonistic regulation by hepatocyte nuclear factor $4 \mathrm{alpha}$ and chicken ovalbumin upstream promoter transcription factor II. J Biol Chem 283:30970-30979

28. Vigliotta G, Miele C, Santopietro S et al (2004) Overexpression of the ped/pea-15 gene causes diabetes by impairing glucose-stimulated insulin secretion in addition to insulin action. Mol Cell Biol 24:5005-5015

29. Xie Z, Dong Y, Scholz R, Neumann D, Zou MH (2008) Phosphorylation of LKB1 at serine 428 by protein kinase $\mathrm{C}$-zeta is required for metformin-enhanced activation of the AMP-activated protein kinase in endothelial cells. Circulation 117:952-962

30. Song P, Xie Z, Wu Y, Xu J, Dong Y, Zou MH (2008) Protein kinase Czeta-dependent LKB1 serine 428 phosphorylation increases LKB1 
nucleus export and apoptosis in endothelial cells. J Biol Chem 283: $12446-12455$

31. Hori H, Sasaoka T, Ishihara H et al (2002) Association of SH2containing inositol phosphatase 2 with the insulin resistance of diabetic $\mathrm{db} / \mathrm{db}$ mice. Diabetes 51:2387-2394

32. Sasaoka T, Wada T, Tsuneki H (2006) Lipid phosphatases as a possible therapeutic target in cases of type 2 diabetes and obesity. Pharmacol Ther 112:799-809

33. Wada T, Sasaoka T, Funaki M et al (2001) Overexpression of SH2containing inositol phosphatase 2 results in negative regulation of insulin-induced metabolic actions in 3T3-L1 adipocytes via its 5'phosphatase catalytic activity. Mol Cell Biol 21:1633-1646

34. Angulo P (2002) Nonalcoholic fatty liver disease. N Engl J Med 346: $1221-1231$

35. Taniguchi CM, Ueki K, Kahn R (2005) Complementary roles of IRS1 and IRS-2 in the hepatic regulation of metabolism. J Clin Invest 115:718-727

36. Farese RV Jr, Zechner R, Newgard CB, Walther TC (2012) The problem of establishing relationships between hepatic steatosis and hepatic insulin resistance. Cell Metab 15:570-573

37. Mason TM (1998) The role of factors that regulate the synthesis and secretion of very-low-density lipoprotein by hepatocytes. Crit Rev Clin Lab Sci 35:461-487
38. Luna V, Casauban L, Sajan MP et al (2006) Metformin improves atypical protein kinase $\mathrm{C}$ activation by insulin and phosphatidylinositol-3,4,5-(PO4)3 in muscle of diabetic subjects. Diabetologia 49:375-382

39. Sleeman MW, Wortley KE, Lai KM et al (2005) Absence of the lipid phosphatase SHIP2 confers resistance to dietary obesity. Nat Med 11: 199-205

40. Nagata N, Matsuo K, Bettaieb A et al (2012) Hepatic Src homology phosphatase 2 regulates energy balance in mice. Endocrinology 153: 3158-3169

41. Rinella ME, Elias MS, Smolak RR, Fu T, Borensztajn J, Green RM (2008) Mechanisms of hepatic steatosis in mice fed a lipogenic methionine choline-deficient diet. J Lipid Res 49:1068-1076

42. Pickens MK, Yan JS, Ng RK et al (2009) Dietary sucrose is essential to the development of liver injury in the methionine-choline-deficient model of steatohepatitis. J Lipid Res 50:2072-2082

43. Macfarlane DP, Zou X, Andrew R et al (2011) Metabolic pathways promoting intrahepatic fatty acid accumulation in methionine and choline deficiency: implications for the pathogenesis of steatohepatitis. Am J Physiol Endocrinol Metab 300:E402-E409

44. Affò S, Dominguez M, Lozano JJ et al (2013) Transcriptome analysis identifies TNF superfamily receptors as potential therapeutic targets in alcoholic hepatitis. Gut 62:452-460 